\title{
Hybridisation and splitting of a crank angle resolved internal combustion engine model using a mean value intake for real-time performance
}

\author{
Xiaoran Han Alessandro Picarelli Mike Dempsey Romain Gillot \\ Claytex service limited, UK, \\ \{xiaoran.han, alessandro.picarelli,mike.dempsey, romain.gillot\} @claytex.com
}

\begin{abstract}
This paper describes splitting a crank angle resolved three cylinder combustion engine with an air path model and a combustion model. This is to distribute the computational effort on hardware by running models on separate cores to achieve real time capability. Hardware tests show the split models are not able to achieve real time because the thermal dynamics of air path model and combustion model are highly interconnected and computing the models on separate cores will introduce delay and solution can become inaccurate and even infeasible. In order to achieve real time capability while ensuring the results are accurate (2-5\% percent max. error), a new method is proposed, in which instead of running with a complete fluid intake and exhaust model, the combustion model runs with a mean value intake model calibrated for many operating points across the speed-load range. The results show that the combustion model running with mean value intake model is able to produce highly accurate result and real time capability is achievable. By using mean value intake model, calibration effort is significantly reduced compared to purely table based method as the mean value model captures essential dynamics and is able to predict reliably between transition from one operating point to another. The mean value method takes into account Air Fuel Ratio (AFR) dynamics and thus calibration against AFR becomes unnecessary. Comparing to a non-mean value purely table based method, the latter requires calibration at densely scattered operating points in order for the transition between each calibration point to be smooth enough. In calibrating the mean value model a controller is designed to control the dynamics error to zero. This control based method shows high efficiency compared to optimization tools as it does not depend on initial values and iteration process of the calibrating parameters. A function is created to automatically create the tables calibrated. The calibrated mean value intake model is run with a combustion model on a Concurrent test/HiL rig and shows real time capability is achieved with good accuracy. The physical engine model is built in Dymola.
\end{abstract}

Keywords: mean value intake model, split engine model, automated calibration

\section{Introduction}

When running models to achieve real time in hardwarein-Loop applications, sometimes it is desirable to split a larger model into separate cores so that parallel processing features in the hardware can compute models simultaneously. However when models running on separate cores are highly interconnected, i.e. have feedback loops, where outputs of subsystem A are fed into subsystem B whose own outputs are in turn fed back as inputs to subsystem A, communication delays due to sampling within feedback loop can cause inaccuracy or infeasibility of the solutions, depending on the size of the delay or sampling frequency. In order to attain a reasonable accuracy of the solution, high frequency sampling is required to reduce the size this delay. However high frequency sampling will create more computational overheads potentially causing more overruns and hence render real time capability unattainable. To avoid feedback loops, a table based or neural network based method or other similar method can be used to replace the subsystems, where the inputs of the table or neural network are command signals at a higher level which does not require feedback from the subsystems. The tables or neural networks are calibrated off-line for each operating point using the inputs and outputs of the subsystems.

When there are lack of dynamical models of a system, the calibration points interval will need to be quite small in order to capture the nonlinear dynamics of the system between each operating point. The densely scattered operating points to be calibrated take a lot of effort to gather in the physical tests and are not always robust and accurate during transient from one operating point to another when simulated. It is therefore desirable to have dynamical models available where the essential dynamics of physical systems can be computed by the models rather than lookup tables. The dynamical models can be calibrated against physical system measurement where the identified parameters of the dynamical system are recorded in look-up tables. Thus the dynamics of the physical system during transition from one operating point to another is captured by the dynamical models. This increases the robustness and accuracy of the calibration as some of dynamical systems can be modeled so that they are robust against certain parameter variations. As a result of the dynamical system 
being robust against parameter variation, this reduces the number of operating points to be calibrated.

For calibration, tools can be used which are gradient based and iterate a number of simulations before convergence criteria is fulfilled. The convergence of gradient based optimization depends on the initial values of the parameters to be calibrated and convergence of the criteria can not always be guaranteed after the end of simulation iterations. For calibration of multiple variables using multiple tunning parameters, where variables are interconnected, choosing initial values of the tunning parameters for convergence becomes even more challenging. This is because the convergence of one variable depends on the convergence of the other variables. Another advantage of having a dynamical model available is that controllers may be designed to ensure convergence of calibrated variables. The inputs of the controllers are the errors between the measured and calibrated variables and outputs of the controllers are tuning parameters. Controller gains can be designed such that errors starting with different initial values will be driven into the neighbourhood of zero. This method only requires one iteration to simulate in the presence of different initial values of calibrated variables, if controllers are designed properly.

Once calibration results are obtained it is time consuming to put them manually into look-up tables. This not only reduces efficiency but can create errors due to wrong data being put into the tables by the user. It can be also hard to debug which data has been wrongly entered to all the calibrated tables if there is an error. This would either require looking through all the tables and checking each of the entries against the calibration result, or creating test experiment to test each table with correct inputs to the table and check if outputs of the table are produced correctly. A more efficient way is to create a function that automatically puts the calibrated results into look-up tables without human intervention. This will increase efficiency and minimize potential user errors.

In this paper, a crank angle resolved three cylinder gasoline engine is considered for testing its real-time performance on a Concurrent real-time test rig. The engine model is required to be split into air path, i.e. intake and exhaust, and combustion using feedback loops so that they can be run on separate cores. It is shown that splitting the model with feedback loops is inefficient in achieving real time performance. Real time performance is jeopardised due to the delay feedback loops and is therefore not achievable with this level of detail. An alternative approach must be considered to eliminate the computational burdens caused by the feedback loops. Motivated by this problem, a look-up table approach becomes a first option for avoiding feedback loops. A mean value intake model is used for generating correct pressure and mass flow rate in intake manifold. Look-up tables are calibrated to generate throttle discharge coefficient and the volumetric efficiency of intake ports that are formulated in mean value intake model. Controllers are designed for the mean value

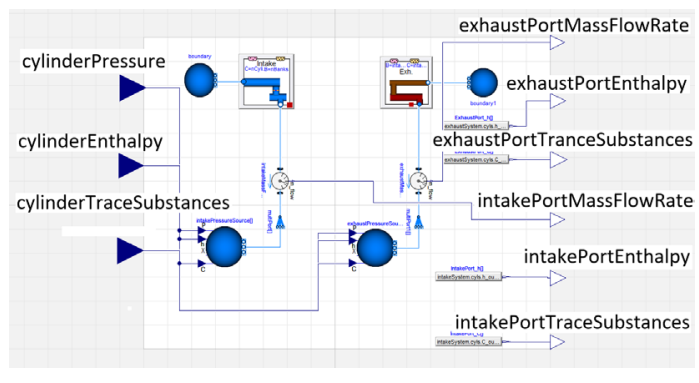

Figure 1. Split air path.

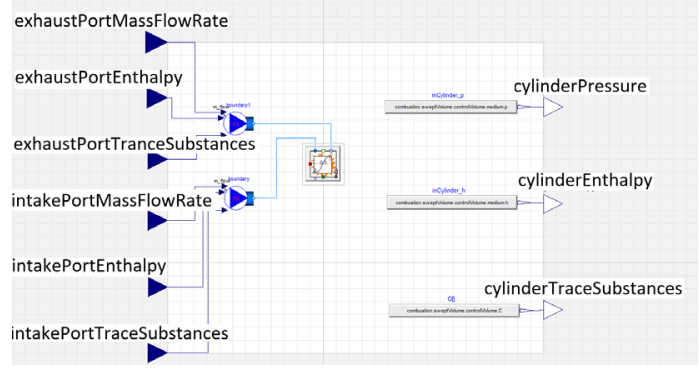

Figure 2. Split combustion.

intake model, whose inputs are mass flow rate and plenum pressure errors between the measurement and values calculated by the mean value model and outputs are the discharge coefficient and volumetric efficiency. It is shown that the mean value intake model calibrated with fewer operating points achieves similar accuracy as those calibrated with more operating points. In addition, the mean value based approach is shown to have much smoother transition phase over using purely table based approach which does not use dynamical models. A function is created to populate the entries of the tables with calibrated results automatically. Because the mean value model takes the effect of air fuel ratio (AFR) into account, calibration can be performed by choosing stoichiometric AFR for different throttle angle, engine speed, intake and exhaust phasing. This AFR dependence reduces calibration for different AFRs since its effect is considered in the model developed. An evaluation of the method, (mean value intake model with look-up tables as a replacement of a physical fluid based intake model with feedback loops to and from the combustion model) is carried out on a Concurrent real-time test rig to show that real-time performance is easily achievable

\section{Splitting engine model with intake and combustion models}

This section shows the splitting of an engine model into an air path model, i.e. intake and exhaust, and a combustion model. Figure 1 and 2 show splitted air path and combustion. Exhaust and intake model output exhaustPortMassFlowRate, exhaustPortEnthalpy, exhaustPortTraceSubstances, intakePortMassFlowRate, intakePortEnthalpy, intakePortTraceSubstances, which are the inputs of combustion model. The outputs of the 
combustion model cylinderPressure, cylinderEnthalpy, cylinderTraceSubstances are the inputs of the air path model. The splitting method is described in detail in (Han, 2017a). The split models are tested on a Concurrent test rig for real-time performance evaluation by running the two models on separate cores. The test results reveal that splitting the models with feedback loops and running the models on separate core will slow down the simulation speed significantly and real time performance is not achievable. An excessively large sampling rate has to be chosen which can cause models to no longer respond correctly. In the following section, a mean value engine intake manifold model is introduced which calculate plenum pressure and mass flow rate at the intake port. The model is calibrated and verified so that it replaces the intake model in Figure 1 and calculates intake plenum pressure reliably.

\section{Mean value engine intake model and its calibration using control design}

Mean value engine models have been developed for modelling and control design (Heywood, 1988), (Guzzella and Onder, 2004). An idle control design of a crank angle resolved engine model using sliding mode and mean value engine model is described in (Han, 2017b) and (Han, 2017c). In this application, the model is calibrated at each operating point so that it can be used to replace the intake manifold as shown in figure 1. This section shows how to tune calibration parameters in the mean engine value model using control design.

\subsection{Mean value model of intake mass flow rate and its calibration}

Mass flow through the throttle for naturally aspirated engine can be approximated as (Guzzella and Onder, 2004)

$$
\dot{m}_{a}(t)= \begin{cases}A_{a} \lambda_{C D} \frac{p_{a}}{\sqrt{R T_{a}}} \frac{1}{\sqrt{2}}, & \text { if } \frac{p_{m}(t)}{p_{a}}<0.5, \\ A_{a} \lambda_{C D} \frac{p_{a}}{\sqrt{R T_{a}}} \sqrt{2 \frac{p_{m}(t)}{p_{a}}\left[1-\frac{p_{m}(t)}{p_{a}}\right]}, & \text { else. }\end{cases}
$$

where $A_{a}$ is the fixed full area of the throttle, $\lambda_{C D}$ is throttle discharge coefficient, which needs to be calibrated, $p_{a}$ is the pressure upstream, ambient pressure, $R$ is the ideal gas constant, $T_{a}$ is the temperature upstream, $p_{m}$ is manifold pressure, which is calculated in (5) and $\dot{m}_{a}$ is the air mass flow through the throttle. $\lambda_{C D}$ is tuned to calibrate (1) at each operating speed and load condition against a measured quantity. Calibration tools can be used to adjust $\lambda_{C D}$ at each iteration based on an optimization criteria so that the value of the calculated mass flow rate $\dot{m}_{a}$ is close to the measured mass flow rate, defined as $\dot{m}_{\text {aMeasured }}$. The number of iterations required before the optimization criteria is fulfilled depends on the convergence rate and the initial value of calibration parameters. A more efficient way to tune $\lambda_{C D}$ by using the control method is presented. The method proves to be very efficient as dependence of the convergence on the initial values of parameters is avoided by designing a controller which yields the feedback loop system to be a linear one. Simulation is only required to run once to determine the correct value of the calibrated parameters.

To design the controller, we define the error between measured air mass and calculated air mass through the throttle as

$$
e_{\text {air }}(t)=m_{\text {aMeasured }}(t)-m_{a}(t)
$$

where $e_{\text {air }}$ is the error. A controller can be designed such that

$$
\lambda_{C D}= \begin{cases}\frac{\sqrt{2 R T_{a}}}{A_{a} P_{a}} K_{m} e_{a i r}(t), & \text { if } \frac{p_{m}(t)}{p_{a}}<0.5, \\ \frac{\sqrt{R T_{a}}}{A_{a} p_{a}} \frac{K_{m} e_{a i r}(t)}{\sqrt{2 \frac{p_{m}(t)}{p_{a}}\left(1-\frac{p_{m}(t)}{p_{a}}\right)},}, & \text { else. }\end{cases}
$$

where $K_{m}$ is a positive control gain. Note that there will always be a pressure drop from ambient pressure $p_{a}$ to $p_{m}$ in order for air flowing through throttle and then down to cylinder via intake valves. Substitute (3) into (1), equation (1) becomes

$$
\dot{m}_{a}(t)=K_{m}\left(m_{\text {aMeasured }}(t)-m_{a}(t)\right)
$$

It is easy to see that by choosing an appropriate control gain $K_{m}$, mass flowed through throttle can be controlled such that $e_{\text {air }} \rightarrow 0$ eventually and $\dot{m}_{a} \rightarrow \dot{m}_{\text {aMeasured }}$.

\subsection{Mean value model of intake manifold pres- sure and its calibration}

Manifold pressure can be modeled as

$$
\dot{p}_{m}(t)=\frac{R T_{m}}{V_{m}}\left[\dot{m}_{a}(t)-\dot{m}_{\beta}(t)\right]
$$

where $T_{m}$ is the manifold temperature, $V_{m}$ is the volume of intake manifold, $\dot{m}_{a}$ is defined in (1) and $\dot{m}_{\beta}$ is the mass flow rate into cylinder. Mass flow rate into cylinder can be modeled as

$$
\begin{gathered}
\dot{m}_{\beta}(t)=\frac{\dot{m}_{e}(t)}{1+\frac{1}{\lambda(t) \sigma_{0}}}, \\
\dot{m}_{e}(t)=\frac{p_{m}(t)}{R T_{m}(t)} \lambda_{l}\left(p_{m}(t), \omega_{e}(t)\right) V_{d} \frac{\omega_{e}(t)}{4 \pi}
\end{gathered}
$$

where $\lambda_{l}(\cdot)$ is the volumetric efficiency of intake ports and valves, denoted as

$$
\lambda_{l}\left(\omega_{e}(t), p_{m}(t)\right)=\frac{m_{\beta}(t)}{\rho_{m}(t) V_{d}}
$$

where $\rho_{m}$ is air density in intake manifold, $V_{d}$ is the engine displacement volume, $\omega_{e}$ is engine speed, $\lambda$ is the air fuel ratio

$$
\lambda(t)=\frac{1}{\sigma_{0}} \frac{\dot{m}_{\beta}(t)}{\dot{m}_{\phi}(t)}
$$




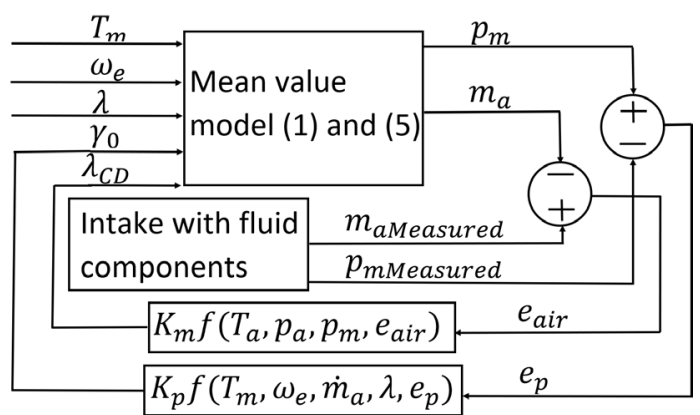

Figure 3. Schematic for control calibration of mean value intake model

where $\sigma_{0}$ is approximately 14.67 and $\dot{m}_{\phi}$ is fuel flow rate. Approximation of the volumetric efficiency of intake ports and valves can be formulated as

$$
\text { where } \quad \begin{aligned}
\lambda_{l}\left(p_{m}(t), \omega_{e}(t)\right) & =\lambda_{l p}\left(p_{m}(t)\right) \lambda_{l w}\left(\omega_{e}(t)\right) \\
\lambda_{l p}\left(p_{m}(t)\right) & =\frac{V_{c}+V_{d}}{V_{d}}-\left(\frac{p_{\text {out }}(t)}{p_{m}(t)}\right)^{\frac{1}{\kappa}} \frac{V_{c}}{V_{d}} \\
\lambda_{l \omega}\left(\omega_{e}(t)\right) & =\gamma_{0}(t)+\gamma_{1} \omega_{e}(t)+\gamma_{2} \omega_{e}(t)^{2}
\end{aligned}
$$

where $p_{\text {out }}$ is the exhaust manifold pressure, $V_{c}$ is clearance volume, and $\kappa, \gamma_{0}, \gamma_{1}$ and $\gamma_{2}$ are tuning parameters. For simplicity, we have chosen $\gamma_{0}$ as calibration parameter and $\gamma_{1}$ and $\gamma_{2}$ are fixed to be small values.

To design a controller for calibrating manifold pressure, the pressure error is defined as

$$
e_{p}(t)=p_{m}(t)-p_{m \text { Measured }}(t)
$$

A controller can be designed such that

$$
\begin{aligned}
\gamma_{0}(t) & =\frac{4 \pi\left(1+\frac{1}{\lambda \sigma_{0}}\right)}{p_{m}(t) \lambda_{l p} V_{d} \omega_{e}(t)}\left(K_{p} V_{m} e_{p}(t)+R T_{m} \dot{m}_{a}(t)\right) \\
& -\gamma_{1} \omega_{e}(t)-\gamma_{2} \omega_{e}^{2}(t)
\end{aligned}
$$

where $K_{p}$ is a positive control gain. Substitute equation (11) into (9) and equation (9) into (6), equation (5) becomes

$$
\dot{p}_{m}(t)=K_{p}\left(p_{m \text { Measured }}(t)-p_{m}(t)\right)
$$

By choosing an appropriate control gain $K_{p}, p_{m}(t) \rightarrow$ $p_{m M e a s u r e d}(t)$ in (12) and $e_{p}(t) \rightarrow 0$. Figure 3 shows a schematic of on using controller design for calibrating mean value intake model, as described in this section.

\subsection{Simulation results}

This section shows calibration performance using controller design described above for $10 \%$ throttle opening, 1000rpm, stoichiometric AFR and default intake and exhaust phasing. Figure 4 shows the measured and calculated mean average and actual value of intake pressure and intake mass flow rate. Figure 5 shows control gains $\gamma_{0}$ and
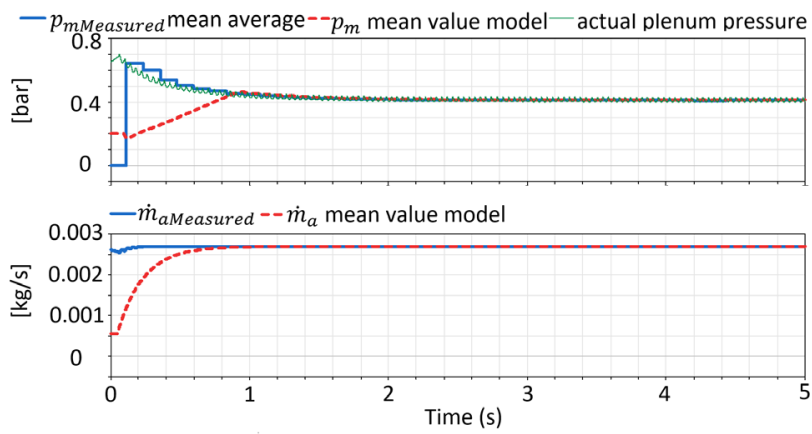

Figure 4. Calibration performance for calculated plenum pressure $p_{m}$ and air flow rate through throttle $\dot{m}_{a}$
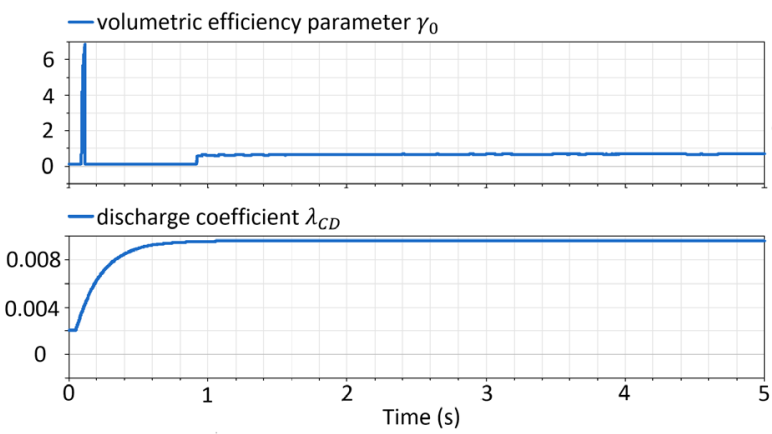

Figure 5. Calibration control gains $\gamma_{0}$ and $\lambda_{C D}$

$\lambda_{C D}$ which are given in (11) and (3). Both calculated intake pressure $p_{m}$ and calculated intake mass flow rate $\dot{m}_{a}$ reach to their corresponding measurement $p_{m \text { measured }}$ and $\dot{m}_{\text {aMeasured }}$ within 2 seconds. Control gains $K_{p}=5$ and $K_{m}=20$ are chosen for computing $\gamma_{0}$ and $\lambda_{C D}$. Thus the proposed method for calibrating mean value air path engine model using control design is shown to be efficient and accurate. Controllers are able to control the calculated values despite their initial values being different from their measured values. The control gains $K_{m}$ and $K_{p}$ can be fixed for all calibrating points because the dynamics of feedback loops for mass flow rate and manifold pressure are only a function of these two gains (4) and (12). Initial values of $m_{a}(t)$ and $p_{m}(t)$ will not have an effect on convergence because the closed loop systems are linear and asymptotic stability of (4) and (12) are guaranteed. This is important in ensuring that calibration process only requires one time simulation for each calibration point and calibration performance can be guaranteed. Due to this feature, calibration process can be automated by running each calibration point sequentially and recording calibration parameters into the entries of look-up tables. It will be difficult to automate this process if optimization methods are used which iterate simulation a number of times until target criterias are met which is not always possible and different initial values of calibration parameters have to be tested, in this case initial values of $\lambda_{C D}$ and $\gamma_{0}$. 


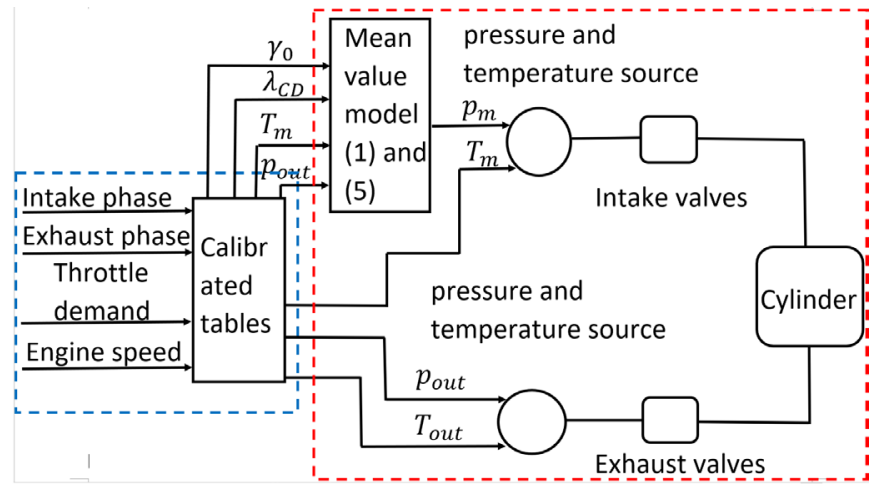

Figure 6. Calibrated tables and mean value model provide pressure temperature source for intake and exhaust valves

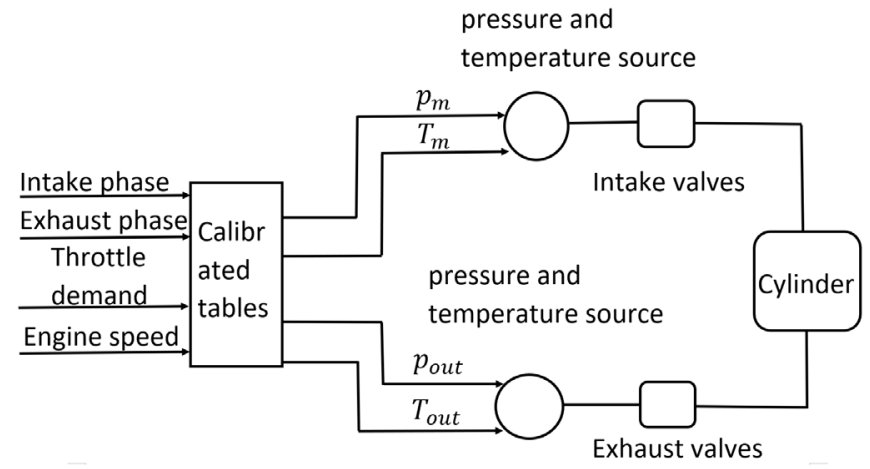

Figure 7. Calibrated tables provide pressure temperature source for intake and exhaust valves

\section{Automation of calibration process}

Automation of the calibration process described in section 3 can be performed by recording all the calibrating parameters into the entries of look-up tables. The model needs to be calibrated against each throttle demand, in take phasing, exhaust phasing, engine speed. Because the mean value model takes into account the AFR values in (6) and (9), only stoichiometric AFR is calibrated. Variation of AFR around stoichiometric will be compensated by the mean value model. The outputs of calibration are throttle discharge coefficient $\lambda_{C D}$ in (3), volumetric efficiency parameters $\gamma_{0}$ in (11), intake manifold temperature $T_{m}$ in (5), exhaust manifold pressure $p_{\text {out }}$ in (9). These four parameters are needed to model mass flow rate $\dot{m}_{a}$ in (1) and intake manifold pressure $p_{m}$ in (5). The manifold pressure $p_{m}$, which is generated by the mean value model, together with calibrated manifold temperature $T_{m}$ can be used to replace the intake manifold in Figure 1. In addition to the four outputs, exhaust manifold temperature, denoted as $T_{\text {out }}$ also needs to be an output so that exhaust manifold in Figure 1 can be replaced by a pressure $p_{\text {out }}$ and temperature $T_{\text {out }}$ source, see Figure 6 . Figure 7 shows calibration without using mean value model. The advantage of using mean value model based calibration over using non-mean value model based calibration will be shown in section 5.1.

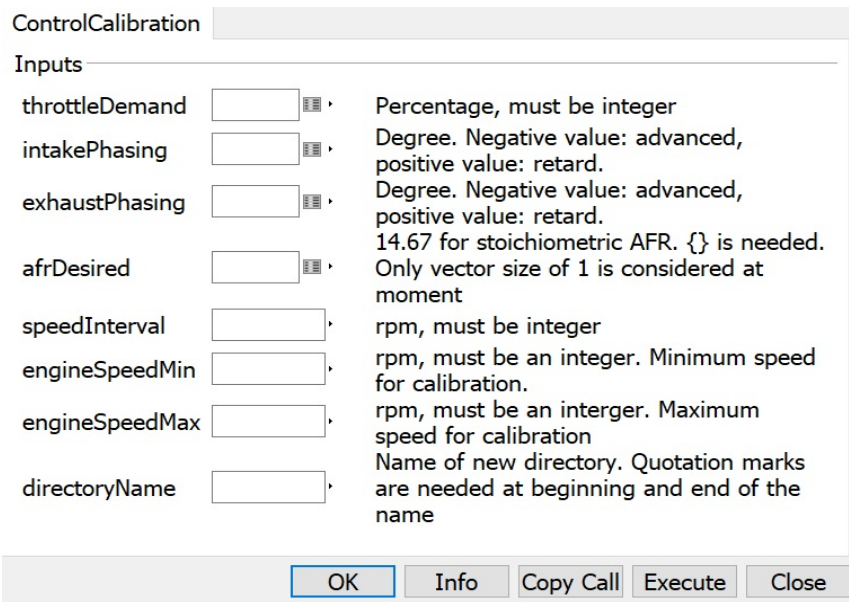

Figure 8. Function for automatic calibration where calibration points can be defined

A function can be created where users can define all the calibration points, Figure 8. For throttle demand, intake and exhaust phase shift, users will need to enter a vector of entries to be calibrated. Because the mean value model in (6) takes into account AFR variations, calibration against a stoichiometric AFR will be sufficient. This is one advantage of using mean value model as it reduces calibration dimensions by one. For engine speed, users will only need to enter minimum and maximum speed and speed interval step between the minimum and maximum speed. If residual of maximum speed over speed interval is not zero, only the last speed before the speed that exceeds the maximum speed by the residual is calibrated. The calibrated result is stored in a directory. Figure 9 shows automatically generated data records, tables, inputs and outputs of the calibrated tables, as shown in blue box in Figure 6 . The number of columns of the tables is always five as it corresponds to the output number of the calibrated table. The number of rows of the tables depends on the engine speed points calibrated. In this case five rows of tables correspond to five engine speed points. The tables are of three dimensions and takes intake phase, exhaust phase and throttle as its three dimensional indexes. The values of calibrated parameters are stored in data records. In such way calibration of four dimensions by use of three dimensional tables can be implemented.

\section{Verification of mean value intake model using simulation result}

This section demonstrates the effectiveness of using a mean value intake model to predict intake manifold pressure. Automatic calibration as described in section 4 is performed to generate required tables. Simulations are carried out for different operating conditions and results are compared between engine models with a fluid component based intake manifold model and a mean value based intake manifold model. To show the advantage of using a mean value model for predicting the manifold pres- 


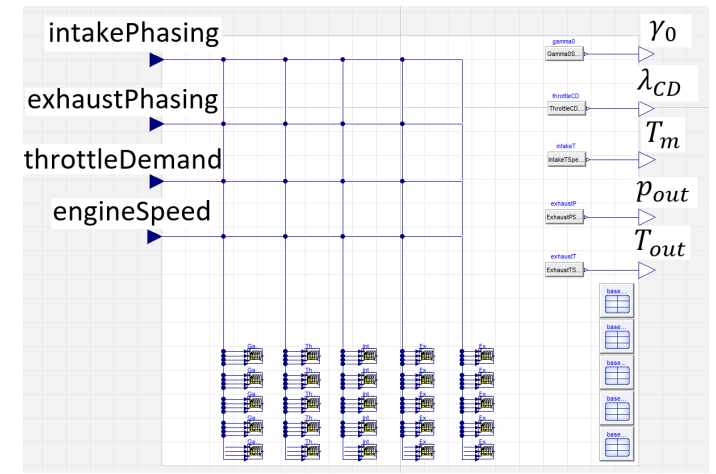

Figure 9. Automatically created calibration tables, data records and calibrating parameters

sure over using tables for predicting the manifold pressure, simulations are performed to show the difference in results. It has been mentioned in section 3 equation (6) and (7) that the mean value model considers the effect of AFR, so that calibration against different AFR is not needed. This feature is demonstrated by using the simulation results. Only one cylinder is used to produce the cylinder pressure. The cylinder pressure is sampled and delayed by the appropriate firing offset to represent the cylinder pressures from the other two cylinders. The cylinder pressures from all three cylinders are then applied to the pistons.

\subsection{Comparison between the fluid based com- ponent intake, the mean value based in- take model and the table based intake model between 5 to 20 percent throttle de- mand and 800 to $1000 \mathrm{rpm}$ engine speed}

The accuracy of the mean value model in predicting plenum pressures is compared with the fluid based component intake model. The advantage of using the mean value based intake model Figure 6 over using a table based intake model Figure 7 is shown in this section. Both mean value model based and table based calibration are performed at the same set of operating points according to Table 1. The actuation inputs for all three experiments, i.e. intake with fluid components, mean value model, tables without mean value model, are shown in Figure 10 to examine the transient performance. Fuel mass injected is fixed for all three experiments so that they are compared under the same fuel amount injected but making use of different intake components.

In Figure 11, the mean plenum pressure of engine models with fluid based component intake is compared with the mean value model based intake and non-mean value model with table based intake. It is shown that the manifold pressure from mean value mode based intake (red dotted) is produced smoothly during transients and matches very well with the pressure from fluid component based intake model (thick blue line). The plenum pressure of the non-mean value model table based intake (thin green line) however behaves more linearly and switches at inter-

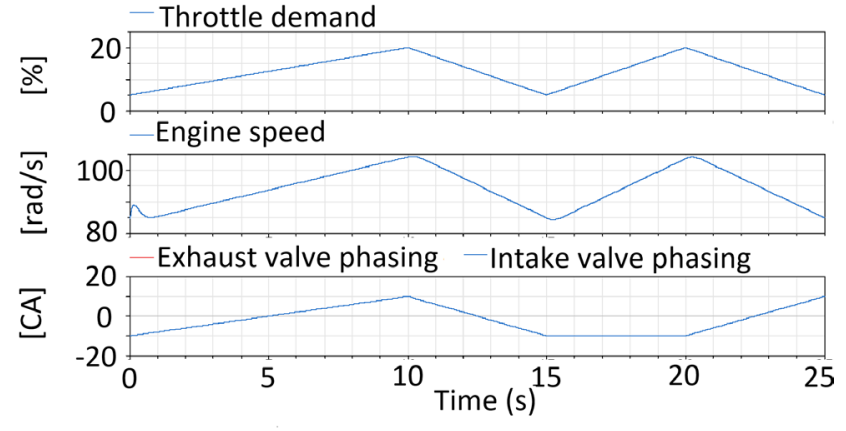

Figure 10. Inputs to calibration tables which are calibrated according to Table 1 with throttle demand 5 to 20 percent and engine speed 800 to $1000 \mathrm{rpm}$

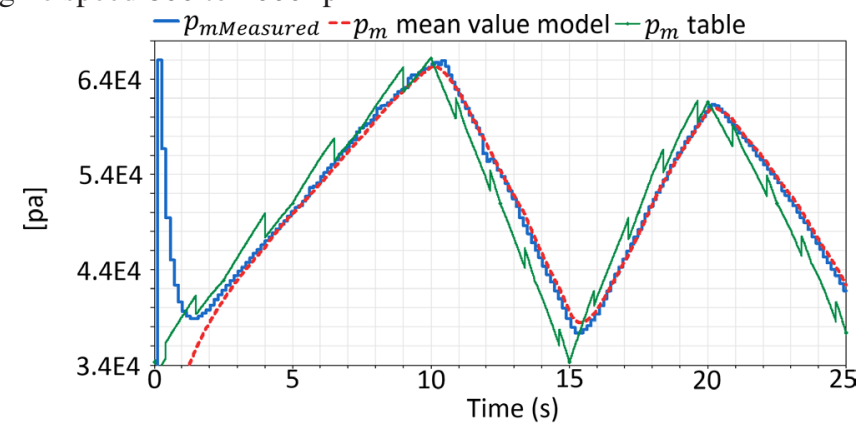

Figure 11. Plenum pressure from engine with fluid component based intake, mean value model based intake and table based intake

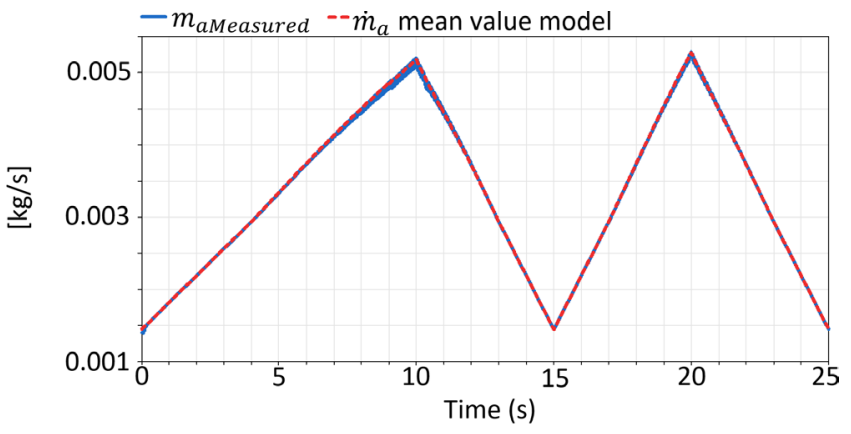

Figure 12. Air flow rate into cylinder with fluid component based intake and mean value model based intake

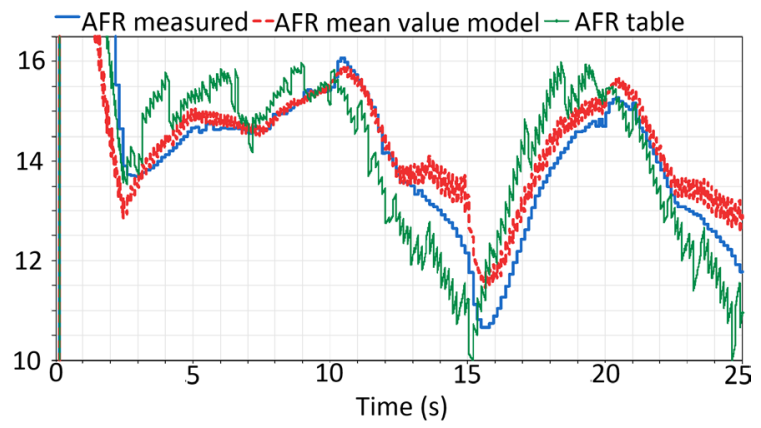

Figure 13. AFR from engine with fluid component based intake, mean value model based intake and table based intake 
Table 1. Calibration points, throttle: 5 to 20 percent; engine speed: 800 to $1000 \mathrm{rpm}$

\begin{tabular}{ll}
\hline Calibration inputs & calibration points \\
\hline Throttle opening (percent) & $5,8,11,14,17,20$ \\
Engine speed (rpm) & $800,850,900,950,1000$ \\
Intake phasing (CA) & $-10,-5,0,5,10$ \\
Exhaust phasing (CA) & $-10,-5,0,5,10$
\end{tabular}

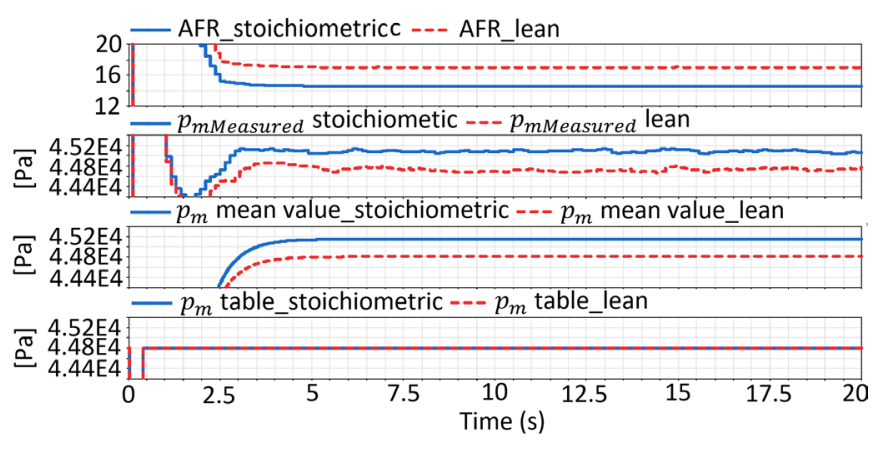

Figure 14. AFR effect on plenum pressure. Subplot 1: AFR values, subplot 2: mean average of plenum pressure from fluid component based intake, subplot 3: plenum pressure from mean value intake, subplot 4: plenum pressure from table based intake

sections between two different calibrated speed profiles. The accuracy is not as good at the result from mean value model. Intake mass flow rates from the fluid based component intake manifold and mean value model based intake manifold are shown in Figure 12. The mass flow rate is calculated by mean value model correctly. Figure 13 shows AFR that corresponds to plenum pressure in Figure 11 and air flow rate in Figure 12. It is shown that the mean value model based intake engine model tracks AFR which follows closely with AFR produced by fluid based component intake engine model, except after 10 second where the AFR from mean value model based intake model is overestimated compared to the AFR produced by the fluid component based intake model. The overestimated AFR is caused by overestimated plenum pressure in the mean value intake model, see Figure 11. To improve calibration precision, more calibration points can be chosen in this region. It is noted that improvement on the accuracy of the calibrated intake manifold temperature will improve the mean value model accuracy during transients. For different AFR values, between a minimum of 11 and a maximum of 16 in Figure 13, the mean value based intake model predict plenum pressures correctly in the presence of AFR variations.

The effect of AFR variation on the mean value model can be further illustrated in Figure 14. The engine is running at 10 percent throttle demand at $900 \mathrm{rpm}$. Intake and exhaust phasing are kept at default. For AFR values from stoichiometric to lean, blue solid to red dashed line in subplot 1 in Figure 14, mean average of plenum pres-
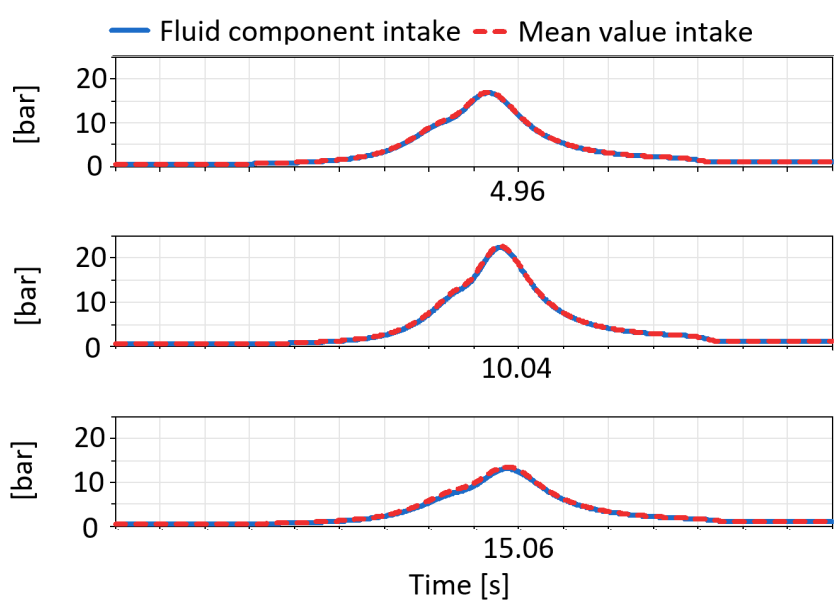

Figure 15. Cylinder pressure between fluid component based intake and mean value model based intake model at 4.96, 10.04 and 15.06 seconds, under plenum pressure in Figure 11

sure in the fluid based component intake model becomes lower, subplot 2 in Figure 14. This trend is reproduced by the mean value model of intake manifold, subplot 3 of Figure 14. The table based non-mean value intake model however produces the same manifold pressure regardless of AFR variations, subplot 4 of Figure 14 .

For the fluid based component intake and mean value model based intake, plenum pressures produced by the two models are shown in Figure 11. Their corresponding cylinder pressures at $5 \mathrm{~s}, 10 \mathrm{~s}, 15 \mathrm{~s}, 20 \mathrm{~s}$ and $25 \mathrm{~s}$ are selected and shown in Figure 15 and Figure 16. It is seen that with a mean value based intake, cylinder pressure at $5 \mathrm{~s}$ and $10 \mathrm{~s}$ matches very closely to cylinder pressure produced using fluid based component intake. At $15 \mathrm{~s}, 20 \mathrm{~s}$ and $25 \mathrm{~s}$ cylinder pressure under mean value based intake is slightly higher than cylinder pressure under fluid component based intake. This is because under the same fuel mass injected the AFR for the mean value based intake is similar at $5 \mathrm{~s}$ and $10 \mathrm{~s}$ but slightly lean at $15 \mathrm{~s}, 20 \mathrm{~s}$ and $25 \mathrm{~s}$ due to more air present in the cylinder, see Figure 13.

\subsection{Comparison between fluid component based intake, mean value based intake model under other throttle demand and engine speed profiles}

This section examines performance of the mean value model based intake under conditions other than calibration points in Table 1.

\subsubsection{Throttle demand from 5 to 70 percent at engine speed from 800 to $1000 \mathrm{rpm}$}

Table 2 shows calibrating points at 5 to 70 percent throttle demand and 800 to $1000 \mathrm{rpm}$ engine speed. The calibrating point interval for throttle demand is 10 percent in Table 2, while it is 3 in Table 1. Calibration interval for intake and exhaust phasing is $10 \mathrm{CA}$ in Table 2, while it is 5 CA in Table 1. This is to examine if the mean value 

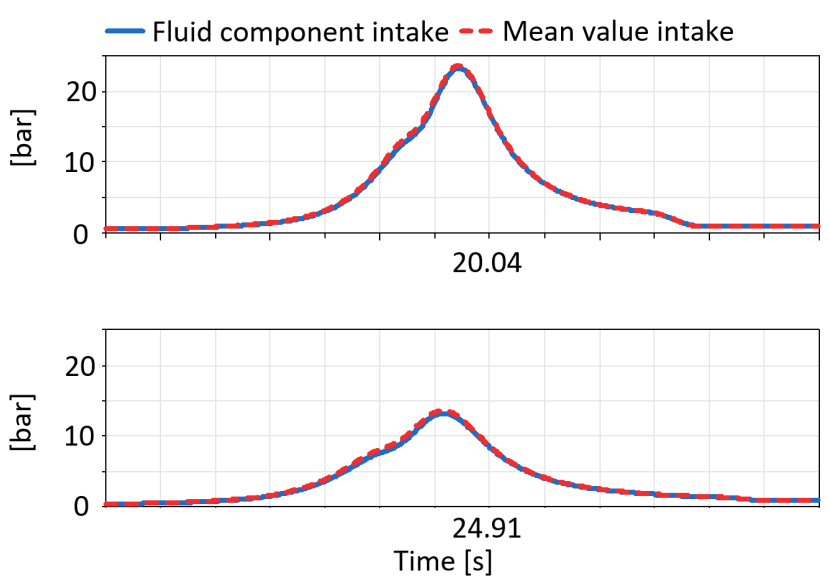

Figure 16. Cylinder pressure between fluid component based intake and mean value model based intake model at 20.04 and 24.91 seconds, under plenum pressure in Figure 11

model is still able to maintain good accuracy despite larger calibrating point intervals.

The actuation inputs for the experiments are shown in Figure 17, which shows different input patterns compared to Figure 10. Plenum pressure and AFR between fluid component based intake and mean value based intake are shown in Figure 18. It is seen that plenum pressure from mean value intake matches very closely to the mean of the plenum pressure produced by fluid based component intake, despite the fact that the mean value model is calibrated with larger calibration intervals. AFR values are similar between 5 and 15 seconds, where AFR changes between rich and lean. After 15 seconds, the AFR from the mean value intake is slightly lower than for the fluid based component intake.

Table 2. Calibration points, throttle: 5 to 70 percent; engine speed: 800 to $1000 \mathrm{rpm}$

\begin{tabular}{lllll}
\hline Calibration inputs & calibration points & & Calibration inputs & calibration points \\
\hline Throttle opening (percent) & $5,10,20,30,40,50,60,70$ & & Throttle opening (percent) & $5,10,+3, \ldots,+3,61$ \\
Engine speed (rpm) & $800,850,900,950,1000$ & & Engine speed (rpm) & $4100,+100, \ldots,+100,5000$ \\
Intake phasing (CA) & $-10,0,10$ & & Intake phasing (CA) & $-10,-6,0,6,10$ \\
Exhaust phasing (CA) & $-10,0,10$ & Exhaust phasing (CA) & $-10,-6,0,6,10$
\end{tabular}

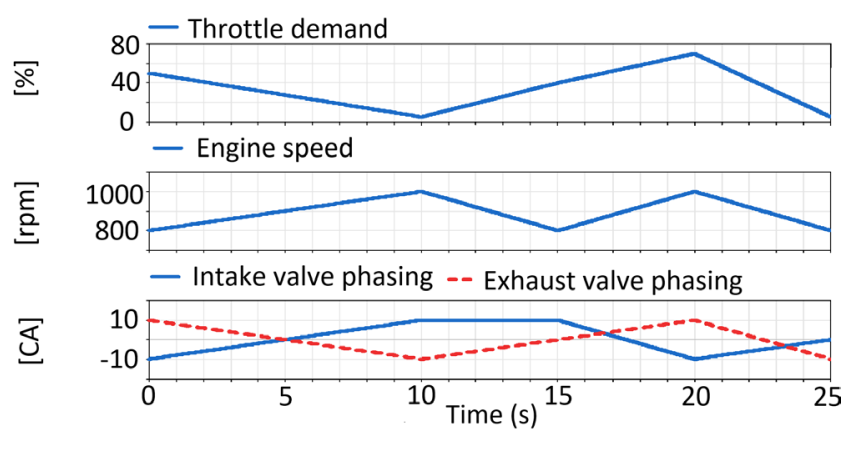

Figure 17. Inputs to calibration tables which are calibrated according to Table 2 with throttle demand 5 to 70 percent and engine speed 800 to $1000 \mathrm{rpm}$

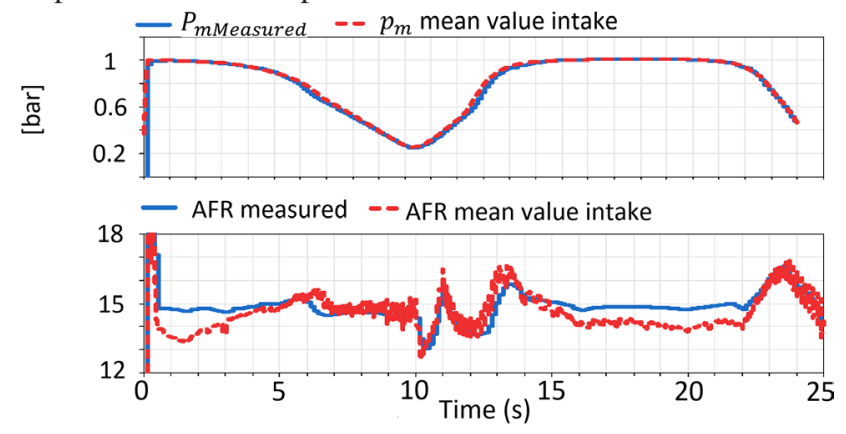

Figure 18. Mean average of plenum pressure and AFR from engine with fluid component based intake and mean value model based intake

mean value model is slightly higher than that produced by the fluid based intake model. This is because the calibration point between 5 and 10 percent throttle demand is not calibrated. This reveals that more throttle demand points should be calibrated for at very low load.

Table 3. Calibration points, throttle: 5 to 60 percent; engine speed: 4100 to $5000 \mathrm{rpm}$

\section{Hardware testing}

\subsubsection{Throttle demand from 5 to 60 percent at engine speed from 4100 to $5000 \mathrm{rpm}$}

Table 3 shows calibration points for the throttle and speed range. Not that for throttle demand, calibration point increases by 3 percent after 10 but between 5 to 10 percent the increment is 5. Figure 19 and 20 show inputs and plenum pressure results. It is noted that the plenum pressure produced by the mean value engine matches very well with plenum pressure produced by fluid based component intake, except at very low load points between 8 and 12 seconds where plenum pressure predicted by the

\subsection{Throttle demand 5 to 20 percent and en- gine speed 800 to $1000 \mathrm{rpm}$}

A quad-core Concurrent test rig, each core having $2.5 \mathrm{GHz}$ clock rate, with 3.9 GB Ram and RedHawk Linux operating system is used for evaluation of the real time performance of the models. Mechanical components (such as the crankshaft) and controller which generates injection, ignition, speed, throttle, intake and exhaust phasing are in one model. The calibrated tables are also within the 


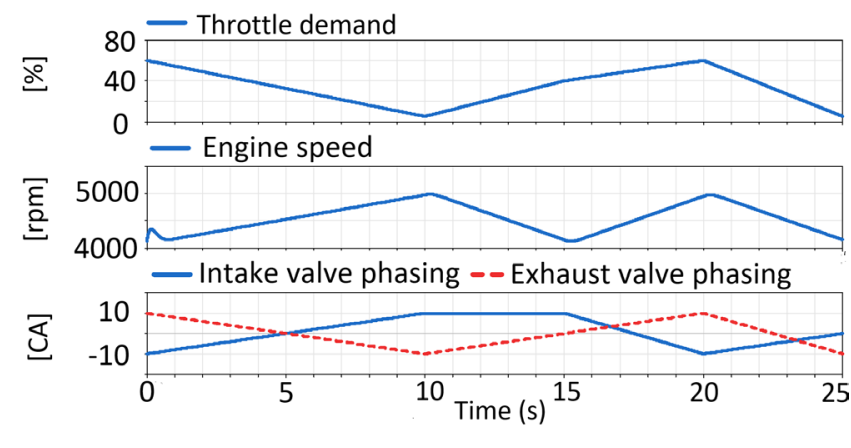

Figure 19. Inputs to calibration tables which are calibrated according to Table 3 with throttle demand 5 to 60 percent and engine speed 4100 to $5000 \mathrm{rpm}$

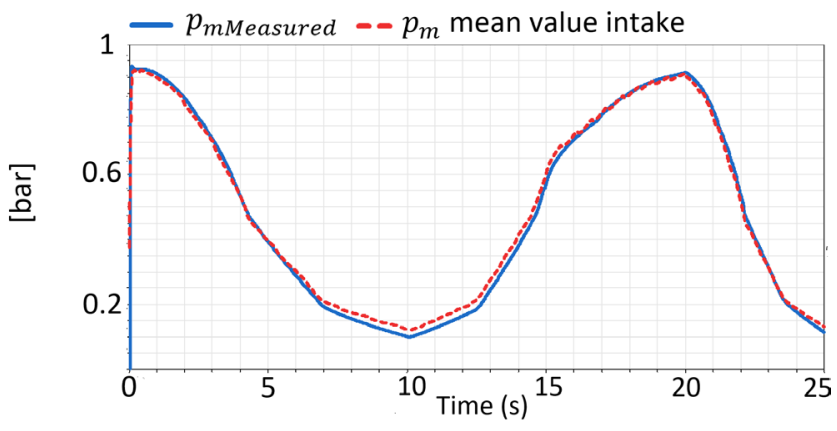

Figure 20. Mean average of plenum pressure from engine with fluid component based intake and mean value model based intake

same model, see blue dashed box in Figure 6. Mean value model, intake and exhaust pressure source, intake and exhaust valves and cylinder are put into another model, see red dashed box in Figure 6. The experiment run on the hardware is the same experiment shown in Figure 10 to Figure 13, where cylinder pressures at different time instants are shown in Figure 15 and Figure 16. Figure 21 to Figure 25 show cylinder pressure recorded on hardware at the same set of time instants studied in Figure 15 and Figure 16. It can be seen that the cylinder pressures produced on the Concurrent rig are of correct values. The execution frames for two models on separate cores are shown in Figure 26, where execution frame equals to 100 micro seconds. It has been tested that the models run in real time with execution frames equal to 500 micro seconds while keeping good accuracy.

\subsection{Throttle demand 5 to 80 percent and en- gine speed 3500 to $6000 \mathrm{rpm}$}

Real time performance can be achieved for the engine running at speed between $3500 \mathrm{rpm}$ to $6000 \mathrm{rpm}$. Figure 27 and Figure 28 show simulation results in Dymola and Concurrent for throttle demand at 65 percent and an engine speed of $5300 \mathrm{rpm}$ by setting the execution frames to be 150 micro seconds. There is a slight fluctuation in the cylinder pressure produced by Concurrent at real time but the accuracy is within a good margin.

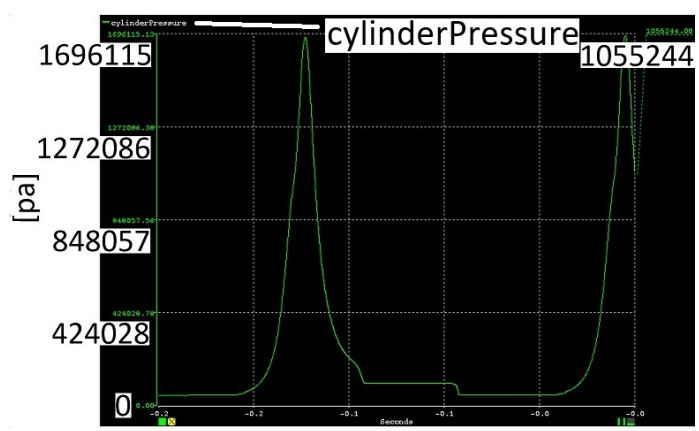

Figure 21. Cylinder pressure at $5 \mathrm{~s}$

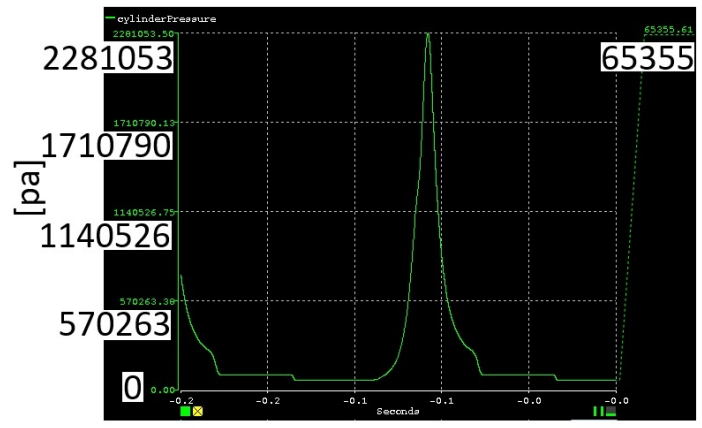

Figure 22. Cylinder pressure at $10 \mathrm{~s}$

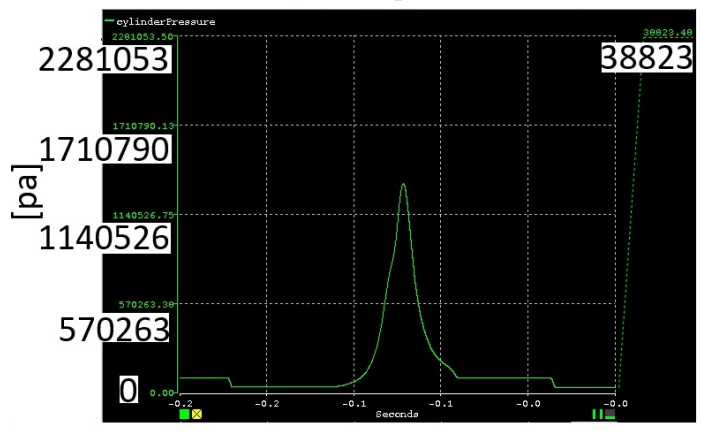

Figure 23. Cylinder pressure at $15 \mathrm{~s}$

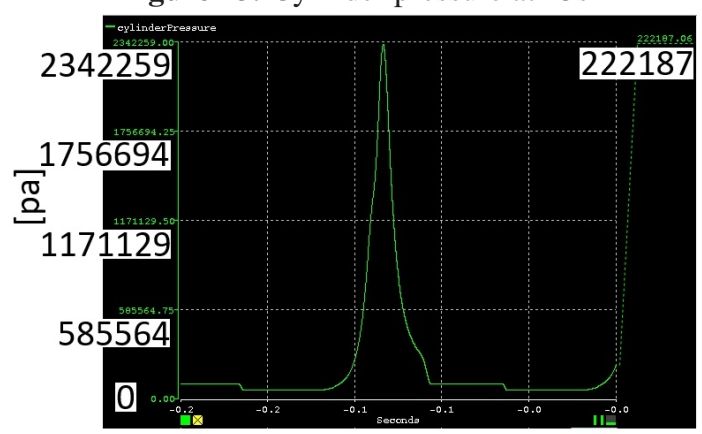

Figure 24. Cylinder pressure at 20s

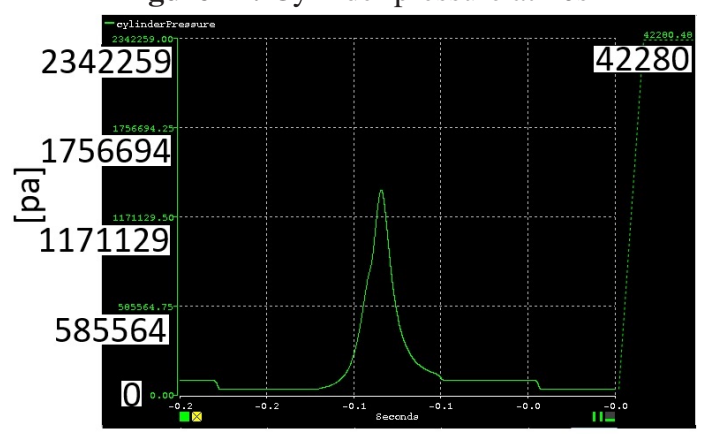

Figure 25. Cylinder pressure at 25s 


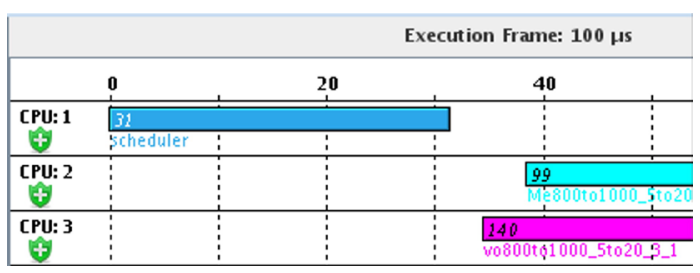

Figure 26. Execution frame for mechanical model on core 2 and cylinder volume model on core 3 at $5 \mathrm{~s}$

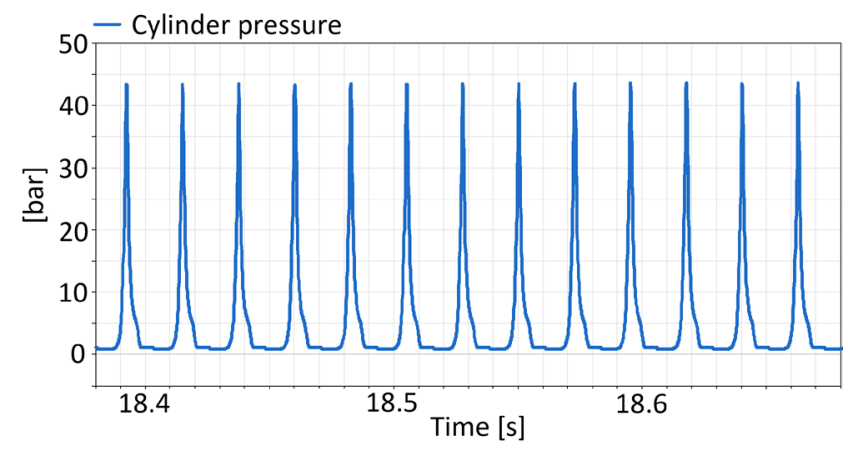

Figure 27. Cylinder pressure at 65 percent throttle demand and $5300 \mathrm{rpm}$ produced in Dymola

\section{Conclusion}

It has been shown that the mean value intake model approach is efficient to be used for crank angle resolved engine simulation in Hardware in Loop testing for real time performance. Automated calibration is efficient and accurate. By using a mean value model intake, calibration against AFR is not needed and the number of dimensional inputs for calibration becomes one less. The result produced by the mean value model is smoother than purely table based methods since plenum pressure is predicted by dynamical models rather than tables.

\section{References}

L. Guzzella and C.H. Onder. Introduction to modelling and control of internal combustion engine systems. Springer-Verlag Berlin Heidelberg, 2004. ISBN 978-3-642-10775-7.

X. Han. Splitting mechanical, fluid ports and thermal ports using real inputs and real outputs. http://www.claytex.com/techblog/splitting-mechanical-and-fluid-devices-using-realinputs-real-outputs/, 2017 a.

X. Han. Linearised Mean Value Engine Model-based Idle Control. http://www.claytex.com/blog/linearised-mean-valueengine-model-based-idle-control/, 2017b.

X. Han. Idle speed control using model based sliding mode control. http://www.claytex.com/blog/idle-speed-control-usingmodel-based-sliding-mode-control/, 2017c.

J.B. Heywood. Internal combustion engine fundamentals. McGraw-Hill, Inc, 1988.

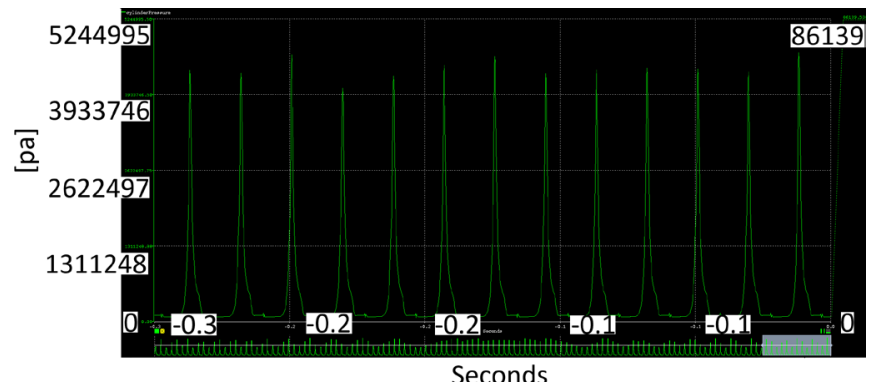

Seconds

Figure 28. Cylinder pressure at 65 percent throttle demand and $5300 \mathrm{rpm}$ produced in Concurrent 